\title{
Aerobes Training für Krebspatienten
}

\begin{abstract}
Onkologen aus Taiwan randomisierten 35 Krebspatienten in zwei Gruppen. Sie wurden entweder wie üblich behandelt oder sie wurden angeleitet zu Hause drei Tage pro Woche ein halbstündiges Gehtraining moderater Intensität zu absolvieren.
\end{abstract}

— Nach acht Wochen zeigte sich, dass sich bei den so trainierten Patienten die Schlafqualität verbessert, die Lebensqualität erhöht und die körperlichen Schmerzen erniedrigt hatten. Die Auto- ren sind daher der Auffassung, dass ein solches häusliches Gehtraining für Krebspatienten zahlreiche, klinisch bedeutsame Vorteile bringt.

\section{Kommentar}

Die Studie ist klein und ist sicher nicht frei von methodischen Schwächen. Dennoch scheinen die Ergebnisse einleuchtend. Das hier geprüfte Gehtraining lässt sich einfach in den Alltag integrieren, es ist nahezu frei von Nebenwirkungen und es ist überaus kostengünstig. Ferner sind die vielfachen positiven Effekte des regelmäßigen aeroben Trainings sehr gut dokumentiert. Daher, so meine ich, ist eine solche Therapie für Krebspatienten, die sie annehmen, wohl einen Versuch wert.

E. ERNST =

- M.-F. Tang, T.-H. Liou, C.-C. Lin

Improving sleep quality for cancer patients: benefits of a home-based exercise intervention. Support. Care Cancer 18 ( 2010) 1329-1399

\section{Autogenes Training gegen Reizdarm}

\section{Mit Selbsthypnose können Reiz- darmpatienten offensichtlich ihre Beschwerden erheblich lindern.}

- Japanische Forscher randomisierten 21 Patienten, die an Reizdarm (Irritable Bowel Syndrome) litten, in zwei Gruppen. Die Experimentalgruppe wurde über acht Wochen mit acht Sitzungen autogenenem Training behandelt, während die Kontrollgruppe lediglich mit allgemeinen Verhaltensmaßnahmen bedacht wurde.

Bei Therapieende gaben $82 \%$ der Experimentalgruppe an, eine ,angemessene Symptomlinderung " (primärer Studienendpunkt) erfahren zu haben. In der Kontrollgruppe lag dieser Prozentsatz bei nur $30 \%$.
Die Differenz war statistisch signifikant. Einige sekundäre Endpunkte zeigten ebenfalls Vorteile für die Therapie mit autogenem Training. Die Autoren schließen aus diesen Daten, dass autogenes Training bei dieser Indikation nützlich sein könnte.

\section{Kommentar}

Autogenes Training ist eine Behandlungsweise, bei der Patienten in kleinen Gruppen einfache Übungen erlernen, die eine Art autohypnotischen Effekt besitzen. Das führt zu einer tiefen Entspannung, die potenziell bei einer Reihe von Erkrankungen nützlich sein könnte. Die klinische Wirksamkeit des autogenen Trainings ist bei einigen Leiden recht gut belegt, z. B. Hypertonie, Angstzuständen oder Schlafstörungen. Beim Reizdarm war die Wirksamkeit des autogenen Trainings bislang nicht erforscht. Die vorliegende Studie hat einige Schwächen, aber ihre Ergebnisse erscheinen dennoch plausibel, nicht zuletzt, da es mehrere Studien gibt, die zeigen, dass Hypnotherapie bei dieser Indikation erfolgreich ist. Da autogenes Training in Gruppen erlernbar und dann selbstständig zu Hause durchführbar ist, hat es den nicht unwesentlichen Vorteil, im Vergleich zur herkömmlichen Hypnotherapie deutlich kostengünstiger zu sein.

E. ERNST =
- M. Shinozaki et al.

Effect of autogenic training on general improvement in patients with irritable bowel syndrome: a randomized controlled trial. Appl. Psychophysiol. Biofeedback 35 (2010) 189-198

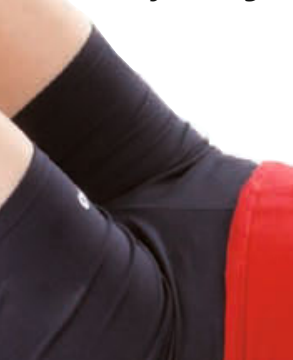

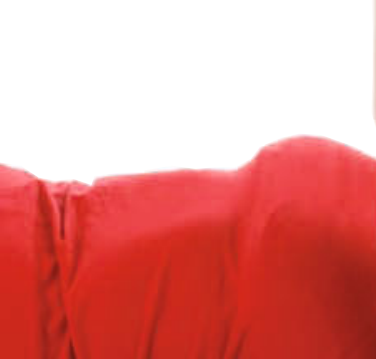

Einfache Übungen besänftigen den Reizdarm.

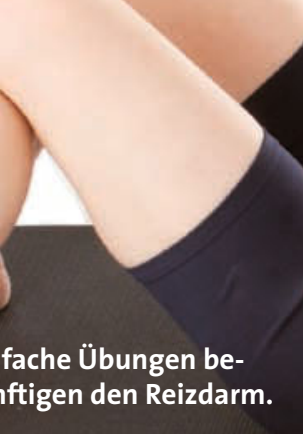

. 